\title{
Liposomal doxorubicin-based treatment in a preclinical model of adrenocortical carcinoma
}

\author{
Constanze Hantel*, Felicitas Lewrick ${ }^{1, *}$, Martin Reincke, Regine Süss ${ }^{1, * *}$ and Felix Beuschlein** \\ Endocrine Research Unit, Medizinische Klinik and Poliklinik IV, Ludwig-Maximilians-University, Ziemssenstraße 1, D-80336 Munich, Germany \\ ${ }^{1}$ Department of Pharmaceutical Technology and Biopharmacy, Albert-Ludwigs University, Sonnenstraße 5, D-79104 Freiburg, Germany \\ (Correspondence should be addressed to F Beuschlein; Email: felix.beuschlein@med.uni-muenchen.de) \\ *(C Hantel and $\mathrm{F}$ Lewrick contributed equally to this work) \\ **(R Süss and $\mathrm{F}$ Beuschlein serve as equally responsible senior authors)
}

\begin{abstract}
Adrenocortical carcinoma (ACC) is a rare endocrine tumor entity with poor prognosis. Medical treatment is limited to common cytotoxic agents, which are associated with low treatment responses. Thus, lack of therapeutic efficacy demands innovative treatment options for patients with advanced ACC. Recently, we have developed and characterized anti-IGF1 receptor (IGF1-R) immunoliposomes (SSLD-1H7) for the treatment of neuroendocrine tumors of the gastroenteropancreatic system. As previous results indicated putative applicability also for other IGF1-Roverexpressing tumor entities, we initiated testing of liposomal preparations in in vitro and in vivo models of ACC. Adrenocortical NCIh295 cells were used for in vitro association studies with different liposomal formulations. Thereby, flow cytometry revealed high cellular association and internalization of anti-IGF1-R immunoliposomes (soy phosphatidylcholine (SPC)/cholesterol (Chol)-polyethyleneglycol (PEG)-1H7, 50·1 $\pm 2 \cdot 2 \%$ ). Moreover, internalization of
\end{abstract}

pegylated liposomes (SPC/Chol-PEG, $57 \cdot 1 \pm 2 \cdot 4 \%$ ) and an even higher uptake of plain liposomes $(84.6$ $\pm 0.8 \% ; P<0.0001)$ were detectable in adrenocortical tumor cells. In vivo, liposomal treatments were investigated on NCIh295 tumor xenografts in pharmacokinetic and therapeutic experiments. A significant reduction in tumor size was detectable in NCIh295 tumor-bearing mice after a single treatment with SSLD-1H7 $(0.89 \pm 0.15 \mathrm{~cm}$; $P=0 \cdot 006)$ and a diminished efficacy for SSLD-PEG + $(1.01 \pm 0.19 \mathrm{~cm} ; P=0.04)$ in comparison with untreated controls $(1.5 \pm 0 \cdot 0 \mathrm{~cm})$. Thus, anti-IGF1-R immunoliposomes have been successfully tested in vitro and in vivo in a preclinical model for ACCs and could, therefore, represent a promising therapeutic approach for this tumor entity. Moreover, a combination of mitotane plus liposomally encapsulated cytostatic agents instead of free drugs could also be an interesting novel treatment option for ACC in the future.

Journal of Endocrinology (2012) 213, 155-161

\section{Introduction}

Adrenocortical carcinoma (ACC) is a rare neoplasm characterized by a dismal prognosis with only $16-38 \%$ of patients surviving more than 5 years after diagnosis (Mansmann et al. 2004, Abiven et al. 2006, Allolio \& Fassnacht 2006). Owing to the absence of specific clinical symptoms, diagnosis and effective treatment can often be greatly delayed. Indeed, most patients with ACC are diagnosed at an advanced stage of disease with large primary tumors, invasion in adjacent organs, and/or metastatic dissemination. Moreover, even after radical resection, as many as $85 \%$ of patients relapse (Allolio \& Fassnacht 2006). Currently, the first prospective randomized interventional trial for ACC (FIRM-ACT, www.firm-act.org) has been finalized to compare streptozotozine plus mitotane with doxorubicin (DXR), etoposide, and cisplatin (EDP) plus mitotane in a cross-over design. However, available data of this trial and from retrospective studies demonstrate that cytotoxic drugs alone have generally produced low response rates (Mansmann et al. 2004, Allolio \& Fassnacht 2006). In addition, the administration of chemotherapeutic drugs is often complicated by dose-limiting side effects (Theodoulou \& Hudis 2004). DXR, for instance, through its small molecular size and amphipathic properties, rapidly distributes to both healthy and diseased tissues (Drummond et al. 1999) and, thus, causes, for example, considerable cardiotoxicity and myelosuppression. Liposomal encapsulation can reduce this toxicity based on the modified biodistribution of the drug (Gabizon et al. 1982). The relatively large size of liposomal carriers (45$200 \mathrm{~nm}$ ) prevents them from passing through the $2 \mathrm{~nm}$ pores found in the endothelium of blood vessels in most healthy tissues or even the $6 \mathrm{~nm}$ pores found in post-capillary venules. Thereby, the efficacy of chemotherapy is enhanced by a resulting increase in drug accumulation in the more porous tumor tissue, an effect that has been described as passive targeting of liposomes (Maeda et al. 2000, Gabizon et al. 2006). 
Further grafting of plain liposomes with polyethyleneglycol (PEG) leads to the formation of a layer on the liposomal surface that protects them from recognition by opsonins. These so-called sterically stabilized liposomes display increased blood stability and, thereby, further enhanced therapeutic efficacy (Huwyler et al. 2008). These mechanisms can be further amplified by active targeting. For this purpose, it is necessary to modify the liposomal surface by coupling a ligand corresponding to a structure of the target tissue. The aim of active targeting is, among other things, to achieve at best a specific internalization by the tumor cells and thereby an effective intracellular access of liposomally encapsulated drugs.

Recently, we initiated the development of a novel antiinsulin-like growth factor 1 receptor (IGF1-R)-coupled liposomal therapy for the treatment of endocrine tumors (Hantel et al. 2010). To achieve this goal, we coupled a monoclonal antibody to the surface of sterically stabilized liposomal DXR (SSLD) for targeting IGF1-R-overexpressing tumor entities and, furthermore, to specifically antagonize downstream signaling ( $\mathrm{Li}$ et al. 1993). As adrenocortical tumorigenesis has been reported to depend on IGF signaling (Logie et al. 1999, Weber et al. 2000, Fottner et al. 2004, Barlaskar et al. 2009), we herein extended our studies in a preclinical model of ACC.

\section{Materials and Methods}

\section{Liposome preparation and coupling procedure}

The basic liposome composition for in vitro analysis consisted of soy phosphatidylcholine (SPC; provided from Lipoid, Ludwigshafen, Germany) and cholesterol (Chol; molar ratio 7:3). For flow cytometry analysis, either $0.5 \mathrm{~mol} \%$ rhodamine-PE (Molecular Probes, Leiden, The Netherlands) or $5 \mathrm{mM}$ FITCdextran 4000 (Sigma-Aldrich) was added to the liposomal formulations, which were prepared as described previously (Hantel et al. 2010). For in vivo experiments, commercially available sterically stabilized liposomes loaded with DXR (SSLD; Caelyx; Essex Pharma GmbH, Munich, Germany) were used. The anti-IGF1-R antibody 1H7 (Acris antibodies, Herford, Germany) as a specific ligand or an unspecific $\operatorname{IgG}$ antibody (Sigma-Aldrich) was coupled to the liposomal surface via sterolbased post-insertion technique using a succinimide-activated sterol-PEG $\mathrm{P}_{1300}$-anchor (sterol-PEG ${ }_{1300}-\mathrm{NHS}$ ), which was synthesized as described previously (Gantert et al. 2008, Hantel et al. 2010). An equivalent amount of untargeted sterol- $\mathrm{PEG}_{1300}$ was added to the SSLD control group (SSLD-PEG +).

\section{Cell culturing and cell experiments}

NCIh295 cells were grown in RPMI-1640 medium as described earlier (Gazdar et al. 1990, Lichtenauer et al. 2008). For association experiments, cells were seeded into 24-well culture plates $24 \mathrm{~h}$ prior to the experiment at a density of $7 \cdot 5 \times 10^{4}$ cells/well. Culture medium was renewed $1 \mathrm{~h}$ prior to the incubation with liposomal preparations $(75 \mathrm{nmol}$ of total lipid of each preparation in $0.5 \mathrm{ml}$ medium) for $2 \mathrm{~h}$ at either $37^{\circ} \mathrm{C}$ or $4{ }^{\circ} \mathrm{C}$. For flow cytometry analysis, cells were washed with $1 \mathrm{ml} \mathrm{PBS}$ and harvested following trypsinization. From each sample, 10000 cells were analyzed using a four-colour FACS Calibur (Becton-Dickinson, Heidelberg, Germany).

\section{Caspase-3/7 assay}

NCIh295 cells were seeded at a density of $4 \times 10^{4}$ cells/well on a 96-well white polystyrene plate (MaxiSorp, Nunc Langenselbold, Germany) and cultivated overnight. After the removal of the medium, cells were treated with medium alone or with medium containing $0 \cdot 25,0 \cdot 5$, or $1 \mu \mathrm{g} / \mathrm{ml} 1 \mathrm{H} 7$ and/or $15 \mu \mathrm{g} \mathrm{DXR} / \mathrm{ml}$ for $8 \mathrm{~h}$. For quantification of caspase3 and caspase-7 activities, the luminescent assay Caspase-Glo 3/7 (Promega) was used. Detection was carried out in a Victor $^{3} 1420$ multi-label counter from Perkin Elmer (Rodgau, Germany) following the manufacturer's protocol.

\section{Cell proliferation ELISA}

NCIh295 cells were seeded at a density of $4 \times 10^{4}$ cells/well on a 96-well plate and cultured overnight. After the removal of the medium, cells were incubated for $48 \mathrm{~h}$ with medium alone or medium containing either $1 \mu \mathrm{g} / \mathrm{ml} 1 \mathrm{H} 7$ and/or $5 \mathrm{ngDXR} / \mathrm{ml}$. For quantification of cell proliferation, a colorimetric immunoassay (Roche) based on the measurement of $5^{\prime}$-bromo- $2^{\prime}$ deoxyuridine (BrdU) incorporation during DNA synthesis was used. Detection was carried out in a SPECTRA microplate reader from Tecan (Crailsheim, Germany).

\section{Animal experiments}

Female athymic NMRI $n u / n u$ mice (6-8 weeks) were purchased from Harlan Winkelmann (Borchen, Germany) and housed under pathogen-free conditions. All experiments were carried out following protocols approved by the Regierung von Oberbayern and in accordance with the German guidelines for animal studies. NCIh295 cells $\left(15 \times 10^{6}\right)$ in a volume of $200 \mu \mathrm{l}$ PBS were inoculated for tumor development subcutaneously into the neck of each mouse. If not stated otherwise, a $10 \mathrm{mg} / \mathrm{kg}$ dose of liposomal DXR (LD, Myocet; Cephalon GmbH, Munich, Germany), SSLD (Caelyx; Essex Pharma GmbH, Munich, Germany), or antibody-coupled SSLD (SSLD-IgG or SSLD-1H7) was administered i.v. after the NCIh295 tumor had been established ( $n=4-5$ mice/group). For pharmacodynamic experiments, $24 \mathrm{~h}$ after the treatment with SSLD-PEG+ and SSLD-1H7, blood samples were taken, tumors were excised, and the samples were analyzed as described before (Hantel et al. 2010). For therapeutic experiments, mice were injected either with LD, SSLD-PEG +, SSLD-IgG, SSLD-1H7, the free antibody $1 \mathrm{H} 7$ alone, or in combination with SSLD-PEG + respectively. Antitumor effects were determined by measuring the longest tumor diameter by 
a calliper gauge. Mice were monitored daily and euthanized when the tumors reached a longest tumor diameter of $15 \mathrm{~mm}$.

\section{Statistical analysis}

All results are expressed as mean \pm S.E.M. Statistical significance was determined using the unpaired $t$-test (Prizm Software, Houston, TX, USA). Statistical significance was defined as $P<0.05$ and is denoted by asterisks $\left({ }^{*} P<0 \cdot 05\right.$; $\left.{ }^{* *} P<0 \cdot 01 ;{ }^{* * *} P<0 \cdot 001\right)$, if not stated otherwise.

\section{Results}

Adrenocortical cellular uptake of liposomal preparations in vitro

Similar to the results previously obtained for other human tumor entities (Hantel et al. 2010), flow cytometry analysis at $37^{\circ} \mathrm{C}$ (Fig. 1A) demonstrated high cellular association of specific $1 \mathrm{H} 7$-coupled liposomes with NCIh295 cells $(50 \cdot 1$ $\pm 2 \cdot 2 \%, P<0 \cdot 0001$ vs controls $0 \cdot 12 \pm 1 \cdot 1)$. While the values at $37^{\circ} \mathrm{C}$ represent passive and active cellular processes, the values at $4{ }^{\circ} \mathrm{C}$ result only from cellular attachment as active metabolic processes do not occur at $4{ }^{\circ} \mathrm{C}$. Thus, the high difference detected between the $4{ }^{\circ} \mathrm{C}(0 \cdot 97 \pm 0)$ and $37^{\circ} \mathrm{C}$ $(50 \cdot 1 \pm 2 \cdot 2)$ values reflected, furthermore, a predominance of active uptake and thereby indicated the cellular internalization of the novel 1H7-liposomes together with fluorescence imaging (Fig. 1B).

Unexpectedly, a comparable internalization of pegylated liposomes $(57 \cdot 1 \pm 2 \cdot 4 \% ; P<0 \cdot 0001$ vs controls and $P=0 \cdot 1$ vs SPC/Chol/PEG-1H7) and an even higher uptake of plain liposomes $(84 \cdot 6 \pm 0.8 \% ; \quad P<0 \cdot 0001$ vs controls and $P=0 \cdot 0001$ vs $\mathrm{SPC} / \mathrm{Chol} / \mathrm{PEG}-1 \mathrm{H} 7)$ were detectable in NCIh295 cells. This phenomenon was absent for cells of other tissue origin such as neuroendocrine tumors of the gastroenteropancreatic system (BON), neuroblastoma (Kelly), breast cancer (MCF7), and prostate carcinoma (DU145; Fig. 1C and as previously described in Hantel et al. (2010)). We utilized SW13 cells as the main other cell line, which has been commonly reported in the literature as a nonsteroidogenic adrenocortical cell line. In fact, SW13 cells also showed significant cellular internalization of plain liposomes. Although the rate was much lower than that observed for NCIh 295 cells, it was clearly higher than tumor cells of non-adrenal origin $(13 \cdot 1 \pm 0 \cdot 1 \% ; \quad P<0 \cdot 0001$ vs controls; Fig. 1C).

To definitively exclude a methodological artifact due to an extraordinary strong extracellular liposomal binding, we increased the stringency of our washing protocols and used specific washing buffers. Even though there was in one case a slight reduction in cellular association, an abrogation of the specific liposomal uptake was not achievable even under the most stringent conditions (Fig. 2A). Furthermore, to eliminate the possibility of a detected artifact related to the applied lipophilic fluorescence marker (rhodamine-PE:

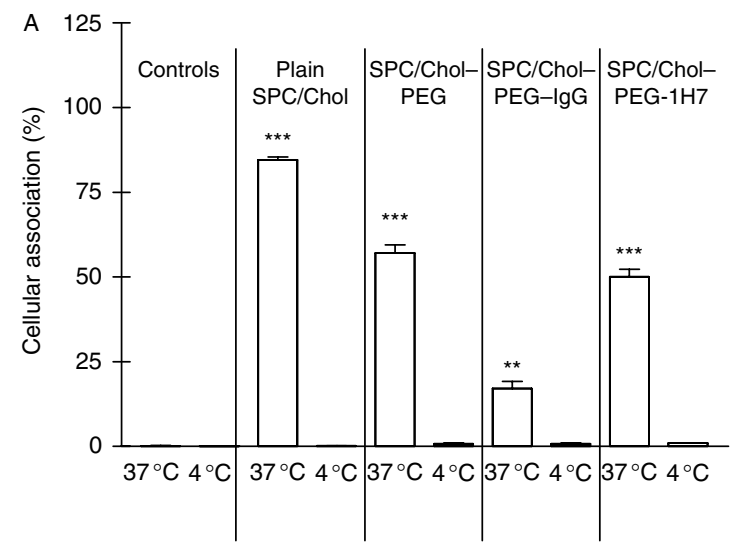

B
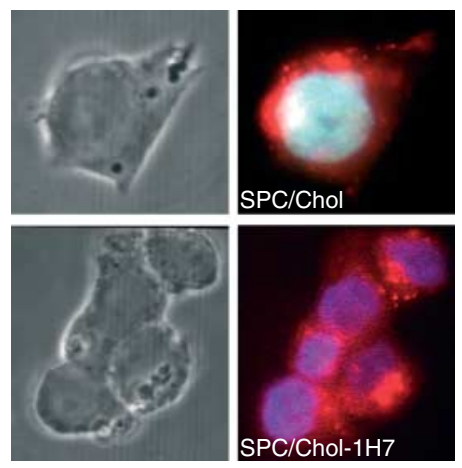

Brightfield

Fluorescence

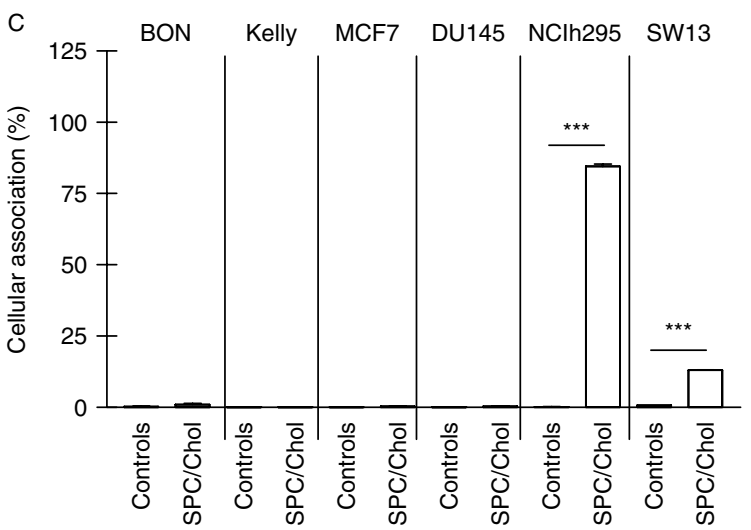

Figure 1 Cellular association of IGF1-R-targeted liposomes (SPC/Chol-PEG-1H7) at $37{ }^{\circ} \mathrm{C}$ and $4{ }^{\circ} \mathrm{C}$ in comparison with the untreated control cells, plain SPC/Chol, SPC/Chol-PEG, and SPC/Chol-PEG-IgG liposomes with NCIh295 cells (A) and fluorescence imaging (B) demonstrating internalization of specifically and non-specifically targeted liposomes in NClh295 cells. Cellular association of plain SPC/Chol liposome preparation in different cell lines demonstrating relevant uptake only in adrenocortical NCIh295 and SW13 cells (C). ${ }^{*} P<0 \cdot 05 ; * * P<0 \cdot 01$; $* * * P<0 \cdot 0001$.

$86 \cdot 5 \pm 0 \cdot 6 \%$ ), association experiments were repeated with water-soluble FITC-dextran encapsulated into the liposomes, which revealed comparable results $(73 \cdot 5 \pm 0 \cdot 8 \%$; Fig. $2 \mathrm{~B})$. 

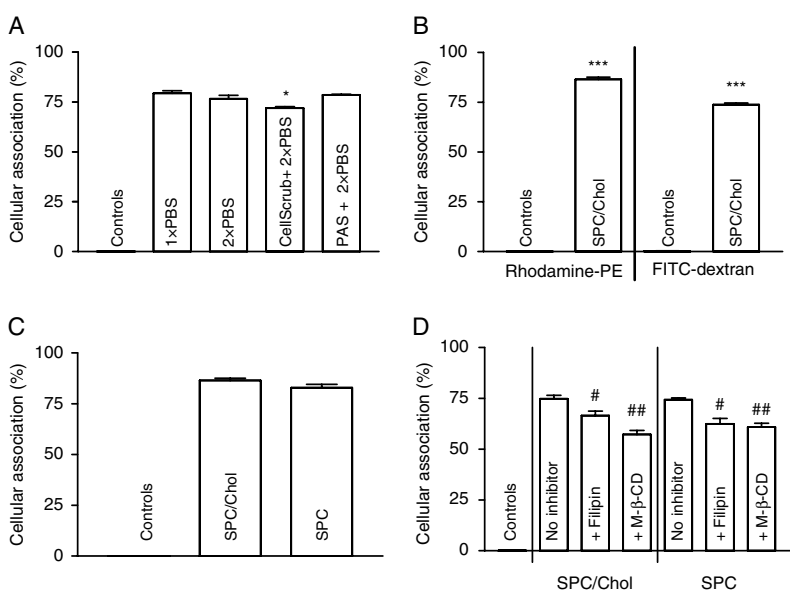

Figure 2 Cellular association of liposomal preparation (SPC/Chol) following washing steps with increasing stringency (A) and influence of different liposomal fluorescence markers (B), cholesterol content $(\mathrm{C})$, and inhibitors of endocytosis $(\mathrm{D})$ on the cellular association of plain liposomes with NClh295 cells. M- $\beta-C D$, methyl- $\beta$-cyclodextrin. Asterisks $\left({ }^{*}\right)$ denote significant differences compared with the controls, while double crosses $\left(^{*}\right)$ represent significant differences over SPC/Chol-treated cells without inhibitor of endocytosis. ${ }^{*} P<0 \cdot 05 ;{ }^{* *} P<0 \cdot 01 ;{ }^{* * *} P<0 \cdot 0001 ;{ }^{*} P<0 \cdot 05$; ${ }^{\#} P<0 \cdot 01 ;{ }^{\# \#} P<0 \cdot 0001$.

We hypothesized that the unusual trapping phenomenon could be related to steroidogenesis and Chol uptake. However, cellular association was independent of liposomal Chol content (Chol-containing preparation: $86 \cdot 5 \pm 0 \cdot 6 \%$, Cholfree preparation: $82 \cdot 9 \pm 1 \cdot 6 \% ; P=0 \cdot 1 ;$ Fig. 2 C). In additional experiments, we inhibited the main uptake routes for naturally occurring lipoproteins such as LDL and HDL. Treatments with inhibitors of caveolae- (filipin) and clathrin (methyl- $\beta$ cyclodextrin)-mediated endocytosis, however, demonstrated only minor effects on cellular association (filipin: SPC/Chol, $66 \cdot 5 \pm 2 \cdot 2 \%, P=0 \cdot 037 ; \quad S P C, 62 \cdot 5 \pm 2 \cdot 7 \%, P=0.024$ vs controls: SPC/Chol, $74 \cdot 8 \pm 1 \%$; SPC, $74 \cdot 4 \pm 0 \cdot 9 \%$; methyl$\beta$-cyclodextrin: SPC/Chol, $57 \cdot 3 \pm 1 \cdot 9 \%, P=0 \cdot 0012$; SPC, $60 \cdot 9 \pm 1 \cdot 8 \%, P=0 \cdot 0028$ vs controls; Fig. $2 \mathrm{D})$.

\section{Antitumor efficacy of DXR and anti-IGF1-R antibody in vitro}

We investigated the anti-tumoral effects of the anti-IGF1-R antibody $1 \mathrm{H} 7, \mathrm{DXR}$, or the combined administration on cell proliferation and apoptosis in NCIh295 cells by BrdU and caspase assays respectively. Induction of apoptosis was evident by an increase in caspase-3/7 activity in comparison with basal values $(100 \cdot 0 \pm 1 \cdot 5 \%) 8 \mathrm{~h}$ upon the $1 \mathrm{H} 7$ and DXR treatments (Fig. 3A). After a single treatment with $250 \mathrm{ng} / \mathrm{ml}$ free $1 \mathrm{H} 7(98.3 \pm 1.9 \% ; P=0.53)$ no significant changes were detectable, while upon incubation with $0.5(111 \pm 1.5 \%$; $P=0 \cdot 007)$ or $1 \mu \mathrm{g} / \mathrm{ml}$ free $1 \mathrm{H} 7(121 \cdot 7 \pm 3 \cdot 2 \% ; P=0 \cdot 004)$, an induction in caspase activity was evident. Similarly, $15 \mu \mathrm{g} \mathrm{DXR} / \mathrm{ml}$ induced a significant increase in apoptosis $(121 \cdot 7 \pm 3 \cdot 2 \% ; P<0 \cdot 0001$ vs basal) and resulted in synergistic therapeutic effects when combined with different concentrations of $1 \mathrm{H} 7(+0.25 \mu \mathrm{g} / \mathrm{ml} \mathrm{1H7:} \mathrm{191.7 \pm 14 \% ;} P=0.003$ vs $1 \mathrm{H} 7$ alone and $P=0 \cdot 23$ vs DXR alone; $+0.5 \mu \mathrm{g} / \mathrm{ml} 1 \mathrm{H} 7$ : $212 \pm 3.5 \% ; P<0 \cdot 0001$ vs both single treatments; $+1 \mu \mathrm{g} / \mathrm{ml}$ 1H7: $221 \cdot 3 \pm 6 \cdot 1 \% ; \quad P<0 \cdot 0001$ vs both single treatments).

Moreover, additive therapeutic effects on cell proliferation were detectable in NCIh295 cells upon combined treatment with $1 \mathrm{H} 7$ and DXR $(65.5 \pm 9 \cdot 2 \% ; P<0.05$ vs untreated; Fig. $3 \mathrm{~B})$, while separate treatments $(1 \mathrm{H} 7: 95 \cdot 5 \pm 6 \cdot 2 \%$; DXR: $104 \cdot 9 \pm 3 \cdot 1 \%)$ did not lead to the inhibition of proliferation compared with untreated cells $(100 \pm 6 \cdot 5 \%)$.

\section{Liposomal blood stability and DXR accumulation in NCIh295 tumors}

DXR blood stability and tumor accumulation were investigated in NCIh295 tumor-bearing mice following the administration of $9 \mathrm{mg} / \mathrm{kg}$ body weight of SSLD-PEG + or SSLD-1H7. Twenty-four hours after a single treatment, we detected significant differences in blood DXR levels $(\mu \mathrm{g} / \mathrm{ml}$ blood; SSLD-PEG+: $4 \cdot 8 \pm 0 \cdot 4$ vs SSLD-1H7: $0 \cdot 8 \pm 0 \cdot 4$; $P<0 \cdot 0001 ;$ Fig. 4A). In accordance with the decrease in blood stability for SSLD-1H7, DXR concentrations in tumor tissues $(\mu \mathrm{g} / \mathrm{g}$ tissue) were significantly lower $(6 \cdot 4 \pm 3 \cdot 1)$ in comparison with those achieved with SSLD-PEG+ (24.5 $\pm 5 \cdot 9$; $P=0.03$ ). In other organs such as spleen, adrenal gland, or liver, no significant differences were evident regarding DXR accumulation upon different treatments (Fig. 4B).

\section{Antitumor efficacy of anti-IGF1-R-targeted immunoliposomes in vivo}

We evaluated the therapeutic efficacy of liposomal preparations in an in vivo ACC model (Fig. 5). Single treatments were initiated for each formulation after subcutaneous NCIh295 tumors of $0.5-0.7 \mathrm{~cm}$ had been established. A significant reduction in tumor size $(\mathrm{cm})$ was detectable from day 4 up to day 32 after a single treatment of tumor-bearing

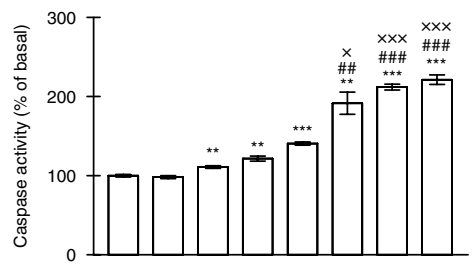

$1 \mathrm{H} 7(\mu \mathrm{g} / \mathrm{ml}) \quad-\quad 0.25 \quad 0.5 \quad 1 \quad-0.250 .5 \quad 1$ $\operatorname{DXR}(\mu \mathrm{g} / \mathrm{ml})$ - $\quad$ - $\quad$ - $\begin{array}{lllll}15 & 15 & 15 & 15\end{array}$

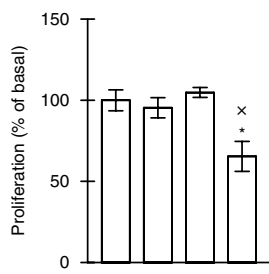

$1 \mathrm{H} 7(\mu \mathrm{g} / \mathrm{ml})-1-1$ $\operatorname{DXR}(\mu \mathrm{g} / \mathrm{ml})-\quad-55$

Figure 3 In vitro effects of DXR and IGF1-R-blocking antibody $(1 \mathrm{H} 7)$ on caspase activity (A) and cell proliferation (B) in human adrenocortical tumor cells (NClh295). Asterisks $\left({ }^{*}\right)$ denote significant differences in comparison with the untreated controls, while double crosses $\left({ }^{\#}\right)$ denote significant differences compared with accordant $1 \mathrm{H} 7$ and crosses $\left({ }^{\times}\right)$denote significant differences compared with single doxorubicin treatments. $* P<0 \cdot 05 ; * * P<0 \cdot 01 ; * * * P<0 \cdot 0001$; ${ }^{\sharp} P<0 \cdot 05 ;{ }^{\# \#} P<0 \cdot 01 ;{ }^{\# \# \#} P<0 \cdot 0001 ;{ }^{\times} P<0 \cdot 05 ;{ }^{\times}{ }^{\prime} P<0 \cdot 01$; $\times \times \times_{P}<0 \cdot 0001$. 
A

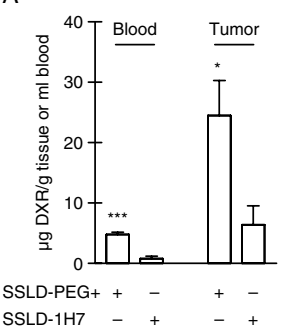

B

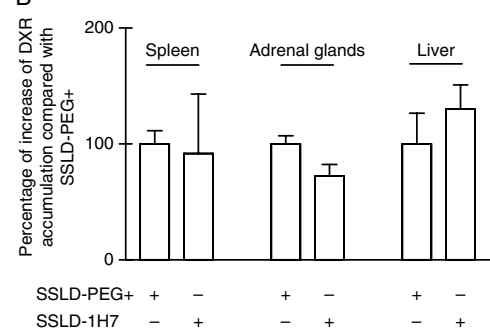

Figure 4 In vivo DXR accumulation in the blood and tumors $24 \mathrm{~h}$ after the treatment of NMRI nu/nu mice bearing NCIh295 xenografts $(n=5)$ with SSLD-PEG + or SSLD-1H7 (A). Comparison of doxorubicin accumulation in different organs upon administration of SSLD-PEG + and $1 \mathrm{H} 7$ antibody-coupled liposomal preparation (B). Asterisks $\left({ }^{*}\right)$ denote significant differences over SSLD-1H7. ${ }^{*} P<0 \cdot 05 ;{ }^{* *} P<0 \cdot 01 ;{ }^{* * *} P<0 \cdot 0001$.

mice with SSLD-1H7. Thereby, therapeutic experiments demonstrated superiority of SSLD-1H7 (day 32: $0 \cdot 89$ $\pm 0 \cdot 15 ; P=0 \cdot 006)$ and to a lesser extent for SSLD-PEG+ $(1 \cdot 01 \pm 0 \cdot 19 ; P=0 \cdot 04)$ in comparison with the untreated controls $(1 \cdot 5 \pm 0)$, while no significant effects were observed for treatments with free $1 \mathrm{H} 7(1 \cdot 38 \pm 0 \cdot 11 ; P=0 \cdot 36)$, LD $(1 \cdot 2 \pm 0 \cdot 3 ; P=0 \cdot 36)$, and unspecific SSLD- $\operatorname{IgG}(1 \cdot 45 \pm 0 \cdot 5$; $P=0 \cdot 36)$ or SSLD-PEG ++ free $1 \mathrm{H} 7(1 \cdot 29 \pm 0 \cdot 17$; $P=0 \cdot 25)$ respectively.

\section{Discussion}

Current first-line polychemotherapeutic regimens for patients with metastatic ACC, such as etoposide, DXR, and cisplatin, achieve mostly partial responses while complete remissions are rarely observed (Allolio \& Fassnacht 2006). Thus, additional treatment options for patients with ACC are urgently needed if surgical treatment options fail. In recent years, drug delivery systems including liposomes have played an emerging role in the field of cancer therapy (Harrington et al. 2002). In general, encapsulation of chemotherapeutic agents results in sustained bioavailability and enhanced drug accumulation in solid tumors (Gabizon 2002). Pegylation of liposomes further improves these pharmacological advantages (Papahadjopoulos et al. 1991). Another strategy to increase drug levels within tumors has been to optimize liposomes for active targeting (Drummond et al. 1999). Recently, we have developed a novel therapeutic approach by coupling an IGF1-R-blocking antibody to liposomal DXR. Herein, we extend these findings from neuroendocrine tumors (Hantel et al. 2010) to adrenocortical cells for which flow cytometry and fluorescence microscopy demonstrates high internalization of 1H7-coupled liposomes. Surprisingly, an even higher uptake of non-antibody-coupled liposomes was evident, which has not been apparent in any other cell line tested so far (Hantel et al. 2010). To gather evidence that our detected results are not based on methodological artifacts, we performed additional experiments and were thereby able

to exclude strong extracellular liposomal binding or extraordinary affinity of NCIh 295 cells to lipophilic rhodamine-PE to explain the detected effect. Thus, these data provided further indirect evidence for a liposomal uptake mechanism that could be specific for adrenocortical cells. As nonsteroidogenic adrenocortical SW-13 cells showed, although at a lower level, the same specific internalization of plain liposomes, we suspected Chol as a possible mediator for liposomal internalization as steroidogenesis requires effective Chol ester uptake by adrenocortical cells. Specifically, LDL particles have been described to be internalized in clathrin-coated pits, while the uptake of HDL occurs dependent on caveolae-mediated endocytosis in liver and steroidogenic tissues (Graf et al. 1999, Connelly \& Williams 2003). However, our experiments showed that the detected uptake was at least not exclusively dependent on caveolae- and clathrin-mediated endocytosis since specific inhibitors were only weakly impeding the investigated effect. Yet, these findings do not exclude other uptake routes such as microvillar channel-dependent mechanisms (Williams et al. 2002).

While the exact mechanism for enhanced liposomal uptake in adrenocortical tumor cells remains uncertain at that point, we proceeded with examination of the antitumor efficacy of DXR and anti-IGF1-R-inhibiting antibody in vitro. Recent studies have provided evidence that combined treatments with cytostatic drugs, in particular with DXR, enhance the efficiency of anti-IGF1-R therapies (Toretsky et al. 1999, Cohen et al. 2005, Martins et al. 2006, Bauer et al. 2007) and we were able to confirm this in vitro for NCIh295 cells. The combination of both substances led to enhanced anti-tumoral effects, suggesting that the combination of these substances in a single immunoliposomal formulation could allow for augmented therapeutic efficacy.

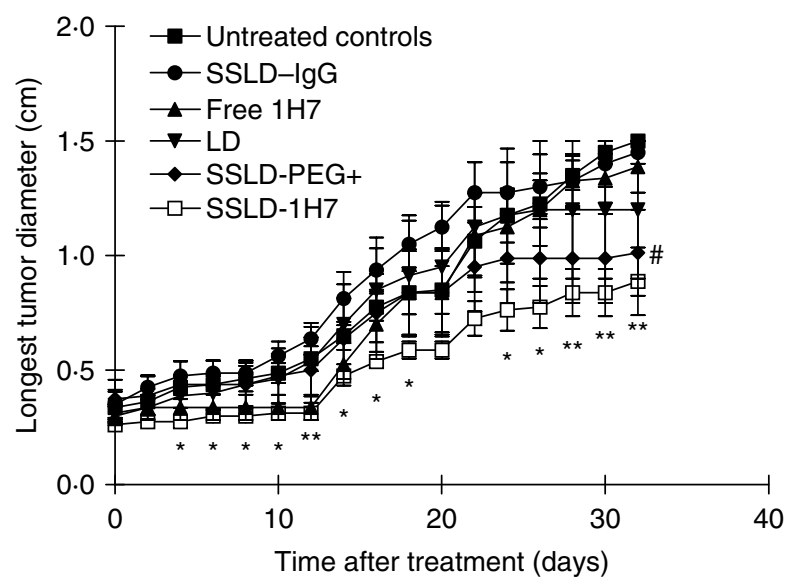

Figure 5 Effects on tumor diameter of NClh295 xenografts in NMRI nu/nu mice after a single treatment with different liposomal preparations $(n=4)$. Asterisks $(*)$ denote significant differences of SSLD-1H7 compared with the untreated controls, while double crosses (\#) represents significant differences of SSLD-PEG + over the untreated controls. ${ }^{*} P<0 \cdot 05 ;{ }^{* *} P<0 \cdot 01 ;{ }^{* * *} P<0 \cdot 0001$. 
Consequently, we proceeded to a more relevant in vivo model. In pharmacokinetic experiments, we tested different liposomal preparations and detected a significantly higher DXR blood stability for non-antibody-coupled SSLDPEG + than that achieved with SSLD-1H7. These findings are in agreement with those previously described for proteinconjugated liposomes (Tardi et al. 1998) and immunoliposomes (Sofou \& Sgouros 2008), and suggest higher immunogeneity and, thereby, faster elimination from the circulation. DXR accumulation in NCIh295 tumors confirmed a significant increase in drug levels after the treatment with SSLD-PEG + compared with SSLD-1H7. However, for this endpoint, it is not possible to discriminate between the effects mediated by blood stability or increased accumulation caused by specific liposomal uptake. Of note, coupling of $1 \mathrm{H} 7$ to the liposomal surface did not lead to an increase in DXR accumulation in other organs such as liver, spleen, or adrenal glands compared with the clinically available liposomal formulation SSLD-PEG + . Therefore, no additional toxic side effects for these organs in comparison with the commercially available formulation in clinical practice would be expected. We next evaluated the therapeutic efficacy of liposomal preparations in vivo. Similar to our previous results in tumor models of neuroendocrine tumors of the gastroenteropancreatic system (GEP-NETs), these experiments confirmed the therapeutic superiority of SSLD-1H7 also for ACC. However, in contrast to GEP-NET cells, an attenuated effect after a single treatment was also evident with SSLD-PEG + in comparison with the untreated controls. On the contrary, treatments with free antibody (1H7), unspecific coupled SSLD-IgG, or DXR-loaded plain liposomes (LD) did not result in significant therapeutic effects. While in vitro experiments had suggested highest efficacy for plain liposomes in adrenocortical cells, for the interpretation of the in vivo studies, additional influences including blood stability have to be accounted for. Pegylation has been shown before to increase blood stability and passive tumor targeting for liposomal DXR in vivo (Drummond et al. 1999). Thus, we assume that a higher uptake of non-pegylated liposomes (LD) is not sufficient to compensate lower blood stability in comparison with pegylated liposomal preparation. Even as SSLD-1H7 showed lower blood stability and DXR accumulation in the tumor tissue in comparison with SSLD-PEG+, we assume further therapeutic effects such as inhibition of IGF1-R signaling together with a more efficient liposomal uptake mechanism and a reduction in IGF1-R presentation on the cell surface (Hantel et al. 2010).

Taken together, SSLD-1H7 has been successfully tested in a preclinical model for ACC and could be regarded as a promising therapeutic approach in the future. Moreover, we provide evidence that ACC tumor cells might represent a particular suitable target for liposomal-based therapeutics in general. A wide range of different chemotherapeutic drugs including DXR, cisplatin, paclitaxel, vincristine, and cytarabine, among others, have been used as liposomal preparations for the treatment of cancer (Drummond et al. 1999). These liposomal encapsulations have been demonstrated to optimize drug release rates by maintaining the blood stability of the formulation and allowing the drug to accumulate at the tumor site. Thus, in recent years, pharmacological tools have been developed and brought into clinical practice that could also improve treatment options for patients with ACC.

An important challenge for the future will be the further characterization of the observed accumulation effect of liposomal preparations by tumor cells of adrenocortical origin and, thereby, the definitive exclusion is that the detected phenomenon is not due to a peculiar functional property with relevance only for the presented cell lines. Thus, to gather further insights into the clinical relevance of the presented findings, future experiments will aim at the development of xenotransplantation models based on surgical tumor samples and their applicability for preclinical studies.

\section{Declaration of interest}

The authors declare that there is no conflict of interest that could be perceived as prejudicing the impartiality of the research reported.

\section{Funding}

This work was supported in part by the Mildred-Scheel-Stiftung (10-2143) to $\mathrm{M} \mathrm{R}$ and R S and Weigand Trust, Germany to F B and C H.

\section{References}

Abiven G, Coste J, Groussin L, Anract P, Tissier F, Legmann P, Dousset B, Bertagna X \& Bertherat J 2006 Clinical and biological features in the prognosis of adrenocortical cancer: poor outcome of cortisol-secreting tumors in a series of 202 consecutive patients. Journal of Clinical Endocrinology and Metabolism 91 2650-2655. (doi:10.1210/jc.2005-2730)

Allolio B \& Fassnacht M 2006 Clinical review: adrenocortical carcinoma: clinical update. Journal of Clinical Endocrinology and Metabolism 91 2027-2037. (doi:10.1210/jc.2005-2639)

Barlaskar FM, Spalding AC, Heaton JH, Kuick R, Kim AC, Thomas DG, Giordano TJ, Ben-Josef E \& Hammer GD 2009 Preclinical targeting of the type I insulin-like growth factor receptor in adrenocortical carcinoma. Journal of Clinical Endocrinology and Metabolism 94 204-212. (doi:10.1210/jc. 2008-1456)

Bauer TW, Fan F, Liu W, Camp ER, Yang A, Somcio RJ, Bucana CD, Singh R \& Ellis LM 2007 Targeting of insulin-like growth factor-I receptor with a monoclonal antibody inhibits growth of hepatic metastases from human colon carcinoma in mice. Annals of Surgical Oncology 14 2838-2846. (doi:10.1245/s10434-007-9486-5)

Cohen BD, Baker DA, Soderstrom C, Tkalcevic G, Rossi AN, Miller PE, Tengowski MW, Wang F, Gualberto A, Beebe JS et al. 2005 Combination therapy enhances the inhibition of tumor growth with the fully human anti-type 1 insulin-like growth factor receptor monoclonal antibody CP-751,871. Clinical Cancer Research 11 2063-2073. (doi:10.1158/ 1078-0432.CCR-04-1070)

Connelly MA \& Williams DL 2003 SR-BI and cholesterol uptake into steroidogenic cells. Trends in Endocrinology and Metabolism 14 467-472. (doi:10.1016/j.tem.2003.10.002)

Drummond DC, Meyer O, Hong K, Kirpotin DB \& Papahadjopoulos D 1999 Optimizing liposomes for delivery of chemotherapeutic agents to solid tumors. Pharmacological Reviews 51 691-743.

Fottner C, Hoeflich A, Wolf E \& Weber MM 2004 Role of the insulin-like growth factor system in adrenocortical growth control and carcinogenesis. Hormone and Metabolic Research 36 397-405. (doi:10.1055/s-2004-814563) 
Gabizon AA 2002 Liposomal drug carrier systems in cancer chemotherapy: current status and future prospects. Journal of Drug Targeting 10 535-538. (doi:10.1080/1061186021000043061)

Gabizon A, Dagan A, Goren D, Barenholz Y \& Fuks Z 1982 Liposomes as in vivo carriers of adriamycin: reduced cardiac uptake and preserved antitumor activity in mice. Cancer Research 42 4734-4739.

Gabizon AA, Shmeeda H \& Zalipsky S 2006 Pros and cons of the liposome platform in cancer drug targeting. Journal of Liposome Research 16 175-183. (doi:10.1080/08982100600848769)

Gantert M, Lewrick F, Adrian JE, Rössler J, Steenpass T, Schubert R \& Peschka-Süss R 2008 Receptor-specific targeting with liposomes in vitro based on sterol-PEG(1300) anchors. Pharmacological Research 26 529-538

Gazdar AF, Oie HK, Shackleton CH, Chen TR, Triche TJ, Myers CE, Chrousos GP, Brennan MF, Stein CA \& La Rocca RV 1990 Establishment and characterization of a human adrenocortical carcinoma cell line that expresses multiple pathways of steroid biosynthesis. Cancer Research $\mathbf{5 0}$ 5488-5496.

Graf GA, Matveev SV \& Smart EJ 1999 Class B scavenger receptors, caveolae and cholesterol homeostasis. Trends in Cardiovascular Medicine 9 221-225. (doi:10.1016/S1050-1738(00)00031-1)

Hantel C, Lewrick F, Schneider S, Zwermann O, Perren A, Reincke M, Süss R \& Beuschlein F 2010 Anti insulin-like growth factor I receptor immunoliposomes: a single formulation combining two anticancer treatments with enhanced therapeutic efficiency. Journal of Clinical Endocrinology and Metabolism 95 943-952. (doi:10.1210/jc.2009-1980)

Harrington KJ, Syrigos KN \& Vile RJ 2002 Liposomally targeted cytotoxic drugs for the treatment of cancer. Journal of Pharmacy and Pharmacology $\mathbf{5 4}$ 1573-1600. (doi:10.1211/0022357002243)

Huwyler J, Drewe J \& Krähenbuhl S 2008 Tumor targeting using liposomal antineoplastic drugs. International Journal of Nanomedicine 3 21-29. (doi:10.2147/IJN.S1253)

Li SL, Kato J, Paz IB, Kasuya J \& Fujita-Yamaguchi Y 1993 Two new monoclonal antibodies against the alpha subunit of the human insulin-like growth factor-I receptor. Biochemical and Biophysical Research Communications 196 92-98. (doi:10.1006/bbrc.1993.2220)

Lichtenauer UD, Shapiro I, Geiger K, Quinkler M, Fassnacht M, Nitschke R, Rückauer KD \& Beuschlein F 2008 Side population does not define stem cell-like cancer cells in the adrenocortical carcinoma cell line NCI h295R. Endocrinology 149 1314-1322. (doi:10.1210/en.2007-1001)

Logie A, Boulle N, Gaston V, Perin L, Boudou P, Le Bouc Y \& Gicquel C 1999 Autocrine role of IGF-II in proliferation of human adrenocortical carcinoma NCI H295R cell line. Journal of Molecular Endocrinology 23 23-32. (doi:10.1677/jme.0.0230023)
Maeda H, Wu J, Sawa T, Matsumura Y \& Hori K 2000 Tumor vascular permeability and the EPR effect in macromolecular therapeutics: a review. Journal of Controlled Release 65 271-284. (doi:10.1016/S0168-3659(99) 00248-5)

Mansmann G, Lau J, Balk E, Rothberg M, Miyachi Y \& Bornstein SR 2004 The clinically inapparent adrenal mass: update in diagnosis and management. Endocrine Reviews 25 309-340. (doi:10.1210/er.2002-0031)

Martins AS, Mackintosh C, Martín DH, Campos M, Hernández T, Ordóñez JL \& de Alava E 2006 Insulin-like growth factor I receptor pathway inhibition by ADW742, alone or in combination with imatinib, doxorubicin, or vincristine, is a novel therapeutic approach in Ewing tumor. Clinical Cancer Research 12 3532-3540. (doi:10.1158/1078-0432. CCR-05-1778)

Papahadjopoulos D, Allen TM, Gabizon A, Mayhew E, Matthay K, Huang SK, Lee KD, Woodle MC, Lasic DD, Redemann C et al. 1991 Sterically stabilized liposomes: improvements in pharmacokinetics and antitumor therapeutic efficacy. PNAS 88 11460-11464. (doi:10.1073/pnas.88.24.11460)

Sofou S \& Sgouros G 2008 Antibody-targeted liposomes in cancer therapy and imaging. Expert Opinion on Drug Delivery 5 189-204. (doi:10.1517/ 17425247.5.2.189)

Tardi P, Bally MB \& Harasym TO 1998 Clearance properties of liposomes involving conjugated proteins for targeting. Advanced Drug Delivery Reviews 32 99-118. (doi:10.1016/S0169-409X(97)00134-8)

Theodoulou M \& Hudis C 2004 Cardiac profiles of liposomal anthracyclines: greater cardiac safety versus conventional doxorubicin? Cancer $\mathbf{1 0 0}$ 2052-2063. (doi:10.1002/cncr.20207)

Toretsky JA, Thakar M, Eskenazi AE \& Frantz CN 1999 Phosphoinositide 3-hydroxide kinase blockade enhances apoptosis in the Ewing's sarcoma family of tumors. Cancer Research 59 5745-5750.

Weber MM, Fottner C \& Wolf E 2000 The role of the insulin-like growth factor system in adrenocortical tumourigenesis. European Journal of Clinical Investigation 30 (Suppl 3) 69-75. (doi:10.1046/j.1365-2362.2000. 0300s3069.x)

Williams DL, Wong JS \& Hamilton RL 2002 SR-BI is required for microvillar channel formation and the localization of HDL particles to the surface of adrenocortical cells in vivo. Journal of Lipid Research 43 544-549.

\section{Received in final form 24 February 2012 \\ Accepted 9 March 2012 \\ Made available online as an Accepted Preprint 9 March 2012}

